ACCEPTED MANUSCRIPT

\title{
Effect of sternal electrode gap and belt rotation on the robustness of pulmonary electrical impedance tomography parameters
}

To cite this article before publication: Louiza Sophocleous et al 2020 Physiol. Meas. in press https://doi.org/10.1088/1361-6579/ab7b42

\section{Manuscript version: Accepted Manuscript}

Accepted Manuscript is "the version of the article accepted for publication including all changes made as a result of the peer review process, and which may also include the addition to the article by IOP Publishing of a header, an article ID, a cover sheet and/or an 'Accepted

Manuscript' watermark, but excluding any other editing, typesetting or other changes made by IOP Publishing and/or its licensors"

This Accepted Manuscript is @ 2020 Institute of Physics and Engineering in Medicine.

During the embargo period (the 12 month period from the publication of the Version of Record of this article), the Accepted Manuscript is fully protected by copyright and cannot be reused or reposted elsewhere.

As the Version of Record of this article is going to be / has been published on a subscription basis, this Accepted Manuscript is available for reuse under a CC BY-NC-ND 3.0 licence after the 12 month embargo period.

After the embargo period, everyone is permitted to use copy and redistribute this article for non-commercial purposes only, provided that they adhere to all the terms of the licence https://creativecommons.org/licences/by-nc-nd/3.0

Although reasonable endeavours have been taken to obtain all necessary permissions from third parties to include their copyrighted content within this article, their full citation and copyright line may not be present in this Accepted Manuscript version. Before using any content from this article, please refer to the Version of Record on IOPscience once published for full citation and copyright details, as permissions will likely be required. All third party content is fully copyright protected, unless specifically stated otherwise in the figure caption in the Version of Record.

View the article online for updates and enhancements. 


\title{
Effect of sternal electrode gap and belt rotation on the robustness of pulmonary electrical impedance tomography parameters
}

\author{
L Sophocleous ${ }^{1}$, AD Waldmann ${ }^{2,3}$, T Becher $^{4}$, M Kallio ${ }^{5}$, M Rahtu ${ }^{5}$ M \\ Miedema $^{6}$, T Papadouri ${ }^{7}$, C Karaoli ${ }^{7}$, DG Tingay $^{8-10}$, A H van Kaam ${ }^{6}, \mathbf{R}$
} Yerworth $^{11}$, R Bayford ${ }^{12}$, I Frerichs ${ }^{4}$

\author{
${ }^{1}$ KIOS Research Centre, Department of Electrical and Computer Engineering, University of Cyprus, \\ Nicosia, Cyprus \\ ${ }^{2}$ Department of Anaesthesiology and Intensive Care Medicine, Rostock University Medical Center, \\ Germany \\ ${ }^{3}$ SenTec AG, Landquart, Switzerland \\ ${ }^{4}$ Department of Anaesthesiology and Intensive Care Medicine, University Medical Centre Schleswig- \\ Holstein, Campus Kiel, Kiel, Germany \\ ${ }^{5}$ PEDEGO Research Unit, University of Oulu and Department of Children and Adolescents, Oulu \\ University Hospital, Oulu, Finland \\ ${ }^{6}$ Department of Neonatology, Emma Children's Hospital, Amsterdam UMC, University of Amsterdam and \\ Vrije Universiteit Amsterdam, Amsterdam, The Netherlands. \\ ${ }^{7}$ Neonatal Intensive Care Unit, Arch. Makarios III Hospital, Nicosia, Cyprus \\ ${ }^{8}$ Neonatal Research, Murdoch Childrens Research Institute, Parkville, Victoría, Australia \\ ${ }^{9}$ Department of Paediatrics, University of Melbourne, Melbourne, Australia \\ ${ }^{10}$ Neonatology, Royal Children's Hospital, Parkville, Australia \\ ${ }^{11}$ Department of Medical Physics and Biomedical Engineering, University College London, London, \\ United Kingdom \\ ${ }^{12}$ Department of Natural Sciences, Middlesex University, London, United Kingdom
}

E-mail: sofokleous.louiza@ucy.ac.cy

Abstract.

Objective: Non-adhesive textile electrode belts offer several advantages over adhesive electrodes and are increasingly used in neonatal patients during continuous electrical impedance tomography (EIT) lung monitoring. However, non-adhesive belts may rotate in unsedated patients and discrepancies between chest circumference and belt sizes may result in a gap between electrodes near the sternum. This project aimed to determine the effects of belt rotation and sternal electrode gap on commonly used lung EIT parameters. Approach: We developed a simulation framework based on a three-dimensional finiteelement model and introduced lung regions with little or no ventilation that could be changed according to a decremental positive end-expiratory pressure (PEEP) trial. Four degrees of sternal gap and belt rotation were simulated and their effect on the EIT parameters silent spaces, centre of ventilation, global inhomogeneity index and overdistension/collapsed lung (OD/CL) analysed. Additionally, seven premature infants were examined to assess the influence of leftward and rightward belt rotations in a clinical setting.

Main results: Small violations of the electrode equidistance criterion and rotations of the belts less than one electrode space exert only minor effects on the EIT parameters and do not impede the interpretation. Rotations of two and three electrode spaces induce nonnegligible effects that might lead to flawed interpretations. The "best PEEP" determined with the OD/CL approach was robust and identifiable with all studied sternal gaps and belt rotations.

Significance: We revealed an important challenge for neonatal EIT applications related to a wide electrode gap at the sternum and belt rotation, which should be avoided in clinical application. 
Keywords: EIT, electrical impedance, respiratory system, electrode rotation, electrode spacing, neonatal lung imaging, EIT belt, electrodes, positive end-expiratory pressure

\section{Introduction}

Minimising ventilator-associated injury to lung tissue in mechanically ventilated neonates is still a challenge in clinical practice (Rocha et al 2018). Electrical impedance tomography (EIT) has been advocated as a novel monitoring tool to help guide lung-protective ventilation (Luepschen et al 2007, Adler et al 2012, Frerichs et al 2017). EIT offers advantages over traditional imaging and monitoring techniques, such as chest radiography and pulse oximetry. An increasing number of studies are demonstrating the potential of EIT monitoring in Neonatal Intensive Care Units (NICU) (Frerichs et al 2001, 2017, Chatziioannidis et al 2011, Miedema et al 2017, Tingay et al 2015, Kallio et al 2019, Hough et al 2016).

EIT is a non-invasive, bedside imaging modality that does not use ionising radiation and provides continuous information on the lung function (Brown et al 1985). EIT has a high temporal resolution (Bayford 2006) and therefore has been widely used as a functional monitoring research tool in a variety of applications in neonates and children (Frerichs et al 2017). Recent clinical studies suggest that EIT can provide useful information to titrate protective positive end-expiratory pressure (PEEP) levels ( Karsten et al 2015, Zhao et al 2019).

During lung EIT monitoring, a very small alternating current is injected at high frequencies through electrodes placed at chest circumference. From the resulting voltages on the surface, the assessment of regional electrical impedance of the tissues within the cross-section under study is made. The placement of individual electrodes around the chest of critically ill patients raises some ergonomic problems, specifically limiting the EIT applications in preterm infants and neonates (Hampshire 1995, Taktak et al 1995, Frerichs et al 2003, Dunlop et al 2006, Miedema et al 2012). A single EIT patient interface could accelerate the implementation of EIT in routine neonatal care (Frerichs et al 2017). Recently a textile interface with 32 electrodes integrated into one belt has been developed (Sophocleous et al 2018, Waldmann et al 2017). This interface offers several advantages in comparison with the use of individual adhesive electrodes. It enables fast, accurate and reproducible positioning of the electrodes around the chest and, in addition, it does not create any discomfort, distress or adverse effects to the neonatal patients (Sophocleous et al 2018). These characteristics offer the potential for use in emergencies such as resuscitation at birth (McCall et al 2017, Tingay et al 2015, 2019a).

An electrode belt allows the exact estimation of the electrode position; however, a limitation exists when it comes to use in neonates. The non-adhesive electrode belt may rotate from its initial position especially during long-term measurements in unsedated patients. Belt rotation may result in inaccurate estimation of electrode position. Although the distance between each electrode and chest circumference is known, forward and inverse models (Boyle et al 2017) may lead to a wrong result or a rotated image. In addition, the belt internal diameter may not exactly match the chest circumference, resulting in a variably sized gap at the point of fixation (closing mechanism) between the first and last electrode over the sternum (Sophocleous et al 2018).

Knowing the exact electrode position is critical for EIT image reconstruction. Albeit absolute imaging is far more sensitive to unequal spacing than difference imaging (Barber and Brown 1988) the latter is still affected when the sensitivity matrix does not accommodate this. Imaging errors from wrong assumptions regarding the electrode positions may impede the clinical interpretation (Lozano et al 1995, Tang et al 2002). It has been shown that the location of the electrode plane 
affects EIT images (Frerichs et al 1999, Karsten et al 2016, Krueger-Ziolek et al 2015, Reifferscheid et al 2011). So far only Karsten et al (2016) has evaluated the impact of different belt positions in the craniocaudal direction on EIT outcomes in a clinical setting, resulting in a proposed optimal thoracic level belt position for adult patients. Braun et al (2018) examined the effect of belt rotation (left/right) on EIT monitoring of hemodynamic parameters in a simulation study. However, there are not yet published data showing the effect of the sternal electrode gap or belt rotation on lung EIT monitoring parameters for the assessment of ventilation.

We aimed to determine the effects of sternal electrode gap and belt rotation on the robustness of commonly used lung EIT parameters. To achieve this goal, the impact of these two effects was analysed using both simulated and clinical neonatal patient data.

\section{Methods}

The method section is divided into two sections. Section 1 addresses the simulation study and Section 2 describes the clinical study.

\subsection{Simulation study}

\subsubsection{Forward model}

For the simulation study, a dedicated three-dimensional finite element model was built: heart, lungs and thorax contour of a healthy female patient in supine position (weight $4.7 \mathrm{~kg}$, gestational age 42 weeks, postmenstrual age 47 weeks) were segmented in a CT image at each intercostal space with the freely available software tool ITK SNAP 3.0 (www.itk.org). The manually segmented organ contours were imported into Matlab (Mathworks, Nantick, MA, USA). The open-source EIDORS EIT image reconstruction platform (Adler et al 2009b) was then used to create a three-dimensional finite element model and 32 rectangular (size $0.5 \times 2 \mathrm{~cm}$ ) electrodes were placed equidistantly on the finite element model along the $5^{\text {th }}$ intercostal space. The sternum of the patient was used as the central reference point, for the electrode arrangement. Electrode 1 was located at the immediate left side of the sternum, whereas electrode 32 was at the right side of the sternum (Figure 1A). To study the influence of electrode distance between electrode 1 and 32 a symmetrical gap was introduced between electrodes 1 and 32, the gap was varied between 0 (equidistant electrode arrangement), 1 (gap of one electrode), 2 (gap of two electrodes) up to 3 (gap of 3 electrodes). For the simulation of gaps 1,2 and 3 all the other electrode distances were kept constant with equal spacing.

To study the influence of belt rotation, the position of electrode 1 was rotated from being at the sternum towards the left side of the model in steps of one electrode up to 3 electrode spaces. 

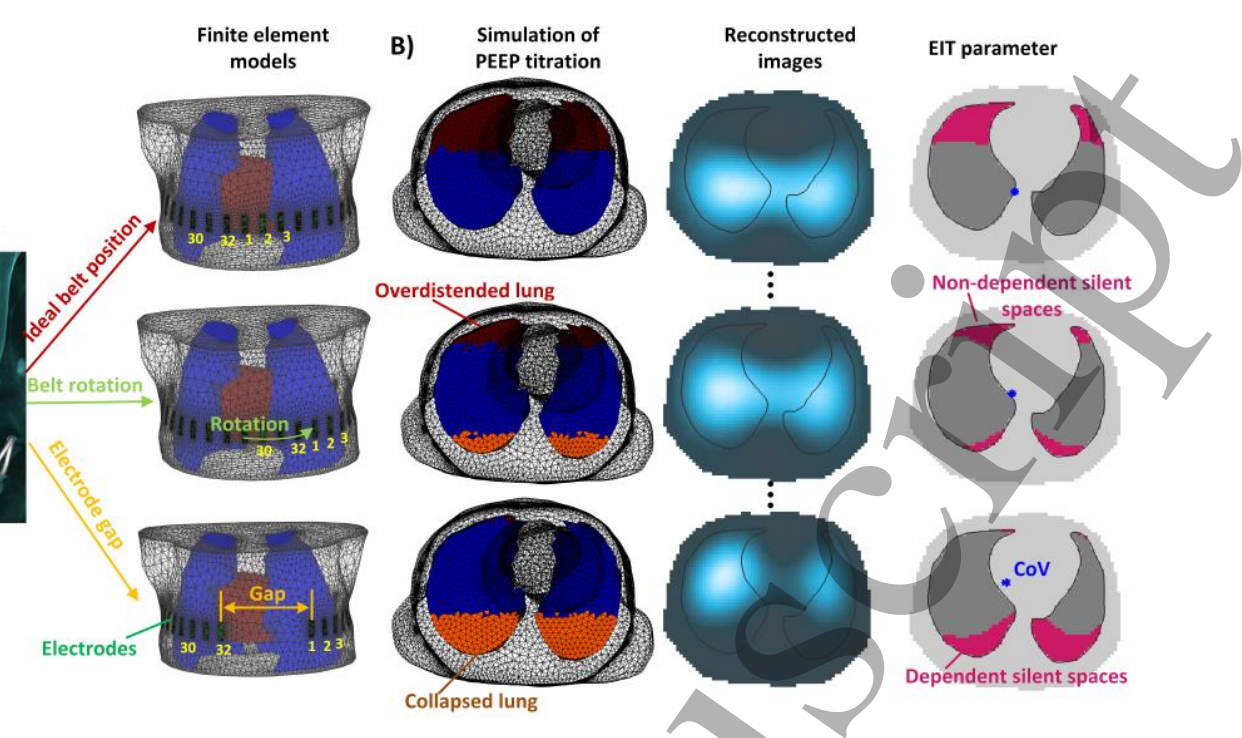

Figure 1 A) The three-dimensional finite element model with lung areas in blue and heart in red, the rectangular electrodes are depicted in dark green. The top finite element model shows the ideal case - electrodes are equally spaced with no gap or rotation, the middle model shows the rotation of the belt to the left and the bottom model the electrode gap. B) Effect of three example PEEP settings: first column shows the introduced overdistended lung regions in dark red, the normally ventilated regions in blue and the collapsed regions in light red colour. The conductivity for the dark red and light red regions was kept constant during ventilation while the conductivity for the blue regions changed. Percentages of overdistended and collapsed regions were introduced in all three dimensions and changed with each positive end-expiratory pressure level. The second column shows the corresponding reconstructed ventilation images, where ventilated areas are shown in bluish and non-ventilated areas in grey colours. In the last column, three example EIT parameters are shown: the blue dot indicates the centre of ventilation $(\mathrm{CoV})$ and the purple areas the areas with little or no apparent ventilation, the so-called Silent Spaces (dependent and non-dependent).

\subsubsection{Simulation protocol}

The manually segmented lung area in the CT scan was divided into three different regions, the first region in the gravity non-dependent ventral area of the lung, representing overdistended lung, the second region in the central part of the lung, representing normally ventilated lung and the last region in the dependent dorsal lung area, representing collapsed lung (Figure 1B). Because of the known effect of the third dimension on EIT data (Rabbani and Kabir 1991) the underlying 3D finite element model contained the collapsed, ventilated and overdistended lung regions in all three dimensions. To mimic conditions under positive end-expiratory pressure (PEEP) ventilation, the size and conductivity of these regions were varied: at high PEEP values the non-dependent lung areas are preferentially likely to be overdistended leading to only small or no ventilation changes, while at low PEEP, collapse predominates in the dependent lung regions with locally reduced or absent ventilation (Costa et al 2009). We simulated the boundary voltages for 11 different PEEP levels, by linearly decrementing PEEP from $13 \mathrm{cmH}_{2} \mathrm{O}$ to $3 \mathrm{cmH}_{2} \mathrm{O}$ in steps of $1 \mathrm{cmH}_{2} \mathrm{O}$. The percentage of overdistension for a PEEP value of $13 \mathrm{cmH}_{2} \mathrm{O}$ was $41 \%$ and decreased gradually to $0 \%$ at $3 \mathrm{cmH}_{2} \mathrm{O}$ while the percentage of collapse for a PEEP value of $13 \mathrm{cmH}_{2} \mathrm{O}$ was $0 \%$ and increased gradually to $47 \%$ at $3 \mathrm{cmH}_{2} \mathrm{O}$.

The conductivity of the introduced overdistended and collapsed areas was set to $0.6 \mathrm{Sm}^{-1}$ and was kept constant during the inspiration, while the conductivity of the rest of the lung was changed from $0.18 \mathrm{Sm}^{-1}$ to $0.05 \mathrm{Sm}^{-1}$ during the inspiration. The conductivities of the heart and thorax were kept constant at $0.6 \mathrm{Sm}^{-1}$ respectively. The conductivity values were selected according to Gabriel et al (1996, 2009). 


\subsubsection{Inverse model}

To map the simulated voltages to a two-dimensional image of $32 \times 32$ pixels, a reconstruction matrix (RM) was calculated in GREIT (Adler et al 2009b) using the following selected values: target-size 0.01 and the noise-figure 0.4. We used uniform background conductivity (Grychtol and Adler 2013, 2014). Difference images were produced by subtracting a reference from the EIT frame sequence taken at the beginning of the breath. The electrode positions were kept constant and neither a gap nor a rotation was introduced in the inverse model.

We calculated the following EIT parameters based on the reconstructed images:

- Centre of ventilation $\left(\mathrm{CoV}_{\mathrm{vd}}\right)$ in the ventral to dorsal direction (Frerichs et al 2006). $\mathrm{CoV}_{\mathrm{vd}}$ describes the distribution of ventilation within lung units across a single plane, and thus assesses the shifts in ventilation in the ventrodorsal direction.

- Areas with little or no apparent ventilation, known as Silent Spaces. These areas are divided into two groups, the non-dependent silent spaces (NSS) representing non-dependent areas of the lungs above a virtual horizontal line passing through $\mathrm{CoV}_{\mathrm{vd}}$ called ventilation horizon and the dependent Silent Spaces (DSS), areas below the ventilation horizon, thus in the dependent part of the lungs (Ukere et al 2016).

- Centre of ventilation $\left(\mathrm{CoV}_{\mathrm{rl}}\right)$ in the right to left direction (Sobota and Roubik 2016). This parameter quantifies the shifts in ventilation distribution from right to left in the horizontal direction.

- Global inhomogeneity index (GI index) which quantifies the overall heterogeneity of tidal volume distribution (Zhao et al 2009).

- The cumulative regional overdistension (OD) and collapse (CL) related to the best compliance as described by Costa et al (2009). This approach yields relative percentages of OD and CL for every PEEP level as well as a "best compromise PEEP" level, where the recruitment of dependent lung areas is maximised and the overdistension of non-dependent lung areas is minimised. This "optimal PEEP" is defined as the intersection between the two lines describing OD and $\mathrm{CL}$.

The parameters $\mathrm{CoV}_{\mathrm{vd}}, \mathrm{CoV}_{\mathrm{rl}}$, GI index and OD/CL were calculated from the entire thorax crosssection whereas DSS and NSS were calculated using the pixels within the anatomical lung region.

\subsection{Clinical study}

This two-centre study was a sub-study of the larger multicentre observational study ( $\mathrm{n}=200$ infants) called Continuous Regional Analysis Device for neonate Lung (CRADL), analysing whether EIT has the potential to optimise respiratory therapy in neonatal and paediatric patients (NCT02962505). The CRADL/ study performed between December 2016 and February 2019 involved 72 hours of continuous EIT recording during routine neonatal intensive care. Infants participating in the CRADL study between April 2018 and February 2019, 44 infants at the Oulu University Hospital, Oulu, Finland (Ethics number: EETTMK 35/2017) and 15 infants at the Arch. Makarios III Hospital, Nicosia, Cyprus (Ethics number: EEBK/EP/2016/32) were considered eligible for our study if they were deemed physiologically stable by the medical team by the end of the CRADL study period. Infants with a body weight less than $600 \mathrm{~g}$, postmenstrual age less than 25 weeks at inclusion, electrically active implants or those suffering from thorax skin lesions were excluded from the study. Prospective informed parental consent was obtained from both parents as part of the CRADL study, and this included manipulation of the EIT electrode belt as detailed below in the two participating centres.

EIT measurements were performed with the CRADL study EIT device (SenTec AG, Landquart, Switzerland). This included the use of a textile 32 electrode belt (Sophocleous et al 2018) circumferentially around the chest at the $5^{\text {th }}$ intercostal space. At the end of the 72-hour CRADL period, the electrode belt was refastened with electrode 1 located $<1 \mathrm{~cm}$ to the left side of the

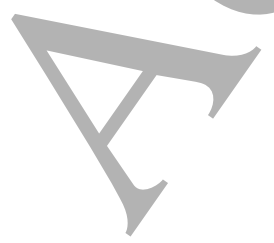


sternum, and a 2-min EIT recording made at 48 frames per second using the same parameters as during the CRADL recording (baseline, or no rotation position). Two-minute recordings were then repeated with the belt rotated with electrode $1,2 \mathrm{~cm}$ to the left and right of the sternum. Neonatal ultrasound gel (Aquasonic 100, Parker Laboratory Inc, USA) was applied to the belt before fastening to ensure optimal skin contact quality was maintained. All measurements were made in the supine position.

Clinical data analysis and processing were performed offline using the SenTec ibeX EIT analysis software package. A sequence of 30 stable inflations, without any failing electrodes, was analysed at each position. The signal was filtered to remove cardiac-related impedance changes. Parameters detailed in 2.1.3 were calculated and analysed. Statistical analysis was performed with IBM SPSS Statistics 25 (IBM Corp., Armonk, NY, USA). Data were compared using repeatedmeasures one-way ANOVA with a Greenhouse-Geisser correction and Bonferroni post hoc test. $p$ values $<0.05$ were considered statistically significant. As this study was part of a different study a feasible sample determined by CRADL study recruitment was used.

\section{Results}

Simulation results

The simulated ventilation maps for each PEEP level, for all three gaps and belt rotations are depicted in Figure 2. The first row of images shows the ideal case, with no gap between electrode 1 and 32, and no belt rotation. The ventilation distribution changes with decreasing PEEP in accordance with gravity-dependent volumetric changes.

At high PEEP levels (13-9 $\mathrm{cmH}_{2} \mathrm{O}$ ) the ventilation is distributed mainly to the dorsal parts of the lungs. With decreasing PEEP the ventilation distribution moves towards ventral lung regions. The introduction of a gap of one electrode barely influences the ventilation distribution, we see comparable results at high PEEP and slight changes to the images at lower PEEP values. At a gap of 2 electrodes, the ventilation distribution is widespread with no clearly defined boundaries, especially in the ventral regions. However, the trend- of more ventilation in the dorsal parts of the lungs at high PEEP and less ventilation at low PEEP remains. At a gap of 3 electrodes, the boundaries between ventilated and non-ventilated areas are less pronounced, furthermore, the left lung is less clear at low PEEP.

Belt rotation of one single electrode leads to comparable results as in the ideal situation with no gap and no rotation. However, a belt rotation of 2 or 3 electrodes creates changes in the image content with clearly identifiable rotated areas showing the ventilated right and left lungs. 
A)

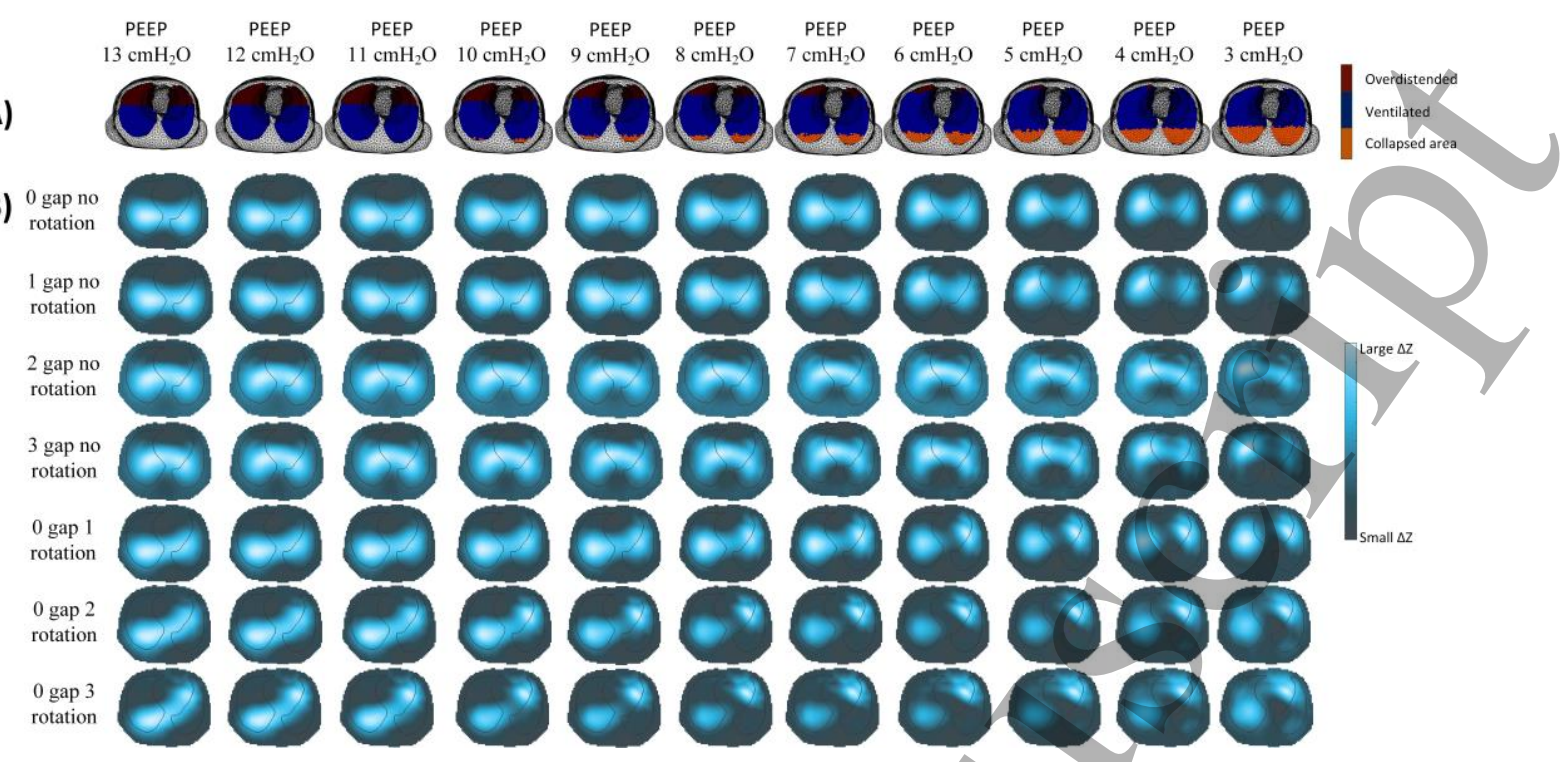

Figure 2 A) Finite element models to simulate the boundary voltages for the 11 simulated positive end-expiratory pressure (PEEP) levels. Overdistended lung regions are shown in dark red, ventilated lung areas in blue and collapsed lung areas in light red. B) Simulation results. The ventilation maps for each PEEP level, electrode gap and rotation are shown. Well ventilated areas are shown in blue and non-ventilated ones in dark grey/colours. The lung contours at the electrode plane are indicated as black lines.

Figure 3 shows the simulation results for EIT parameters at each different simulated PEEP levels, gaps and belt rotations. With no belt gap or rotation at high PEEP levels, there are no DSS until PEEP falls below $10 \mathrm{cmH}_{2} \mathrm{O}$ after which the amount of Silent Spaces increases linearly until a maximum of $25 \%$ at a PEEP of $3 \mathrm{cmH}_{2} \mathrm{O}$. The opposite is true for NSS, which is greatest at a high PEEP and fall to almost zero at a PEEP $3 \mathrm{cmH}_{2} \mathrm{O}$. The $\mathrm{CoV}_{\mathrm{vd}}$ is $56 \%$ at a PEEP of $13 \mathrm{cmH}_{2} \mathrm{O}$ and shifts towards the ventral lung with sequentially lower PEEP levels. The $\mathrm{CoV}_{\mathrm{rl}}$ is exactly in the centre of the thorax $(50 \%)$ at a PEEP of $13 \mathrm{cmH}_{2} \mathrm{O}$ but moves towards the right lung as PEEP is lowered. The GI index was 0.92 at a PEEP of $13 \mathrm{cmH}_{2} \mathrm{O}$ and then decreased linearly towards 0.85 at PEEP $6 \mathrm{cmH}_{2} \mathrm{O}$, indicating a decrease in inhomogeneity. At PEEP levels below $6 \mathrm{cmH}_{2} \mathrm{O}$, inhomogeneity increased again, culminating in a GI index of 0.89 at PEEP $3 \mathrm{cmH}_{2} \mathrm{O}$.

The highest OD and the lowest CL were calculated at a PEEP of $13 \mathrm{cmH}_{2} \mathrm{O}$ (OD: 41\%; CL: $0 \%$ ), with OD decreasing and CL increasing simultaneously at lower PEEP levels. The "crossover point" between the lines was identified at a PEEP of $7 \mathrm{cmH}_{2} \mathrm{O}$ with OD $14.5 \%$ and CL $16.4 \%$. At zero PEEP, OD reached $0 \%$ while CL had increased to $47.3 \%$.

Overall belt rotation has a greater impact on the EIT parameters than electrode gap. The main impact of the electrode rotation was observed in DSS when compared to no rotation, and the degree of impact varied with PEEP levels. A similar impact on $\mathrm{CoV}_{\mathrm{rl}}$ and the GI index, with loss of linearity at PEEP levels was also observed. 

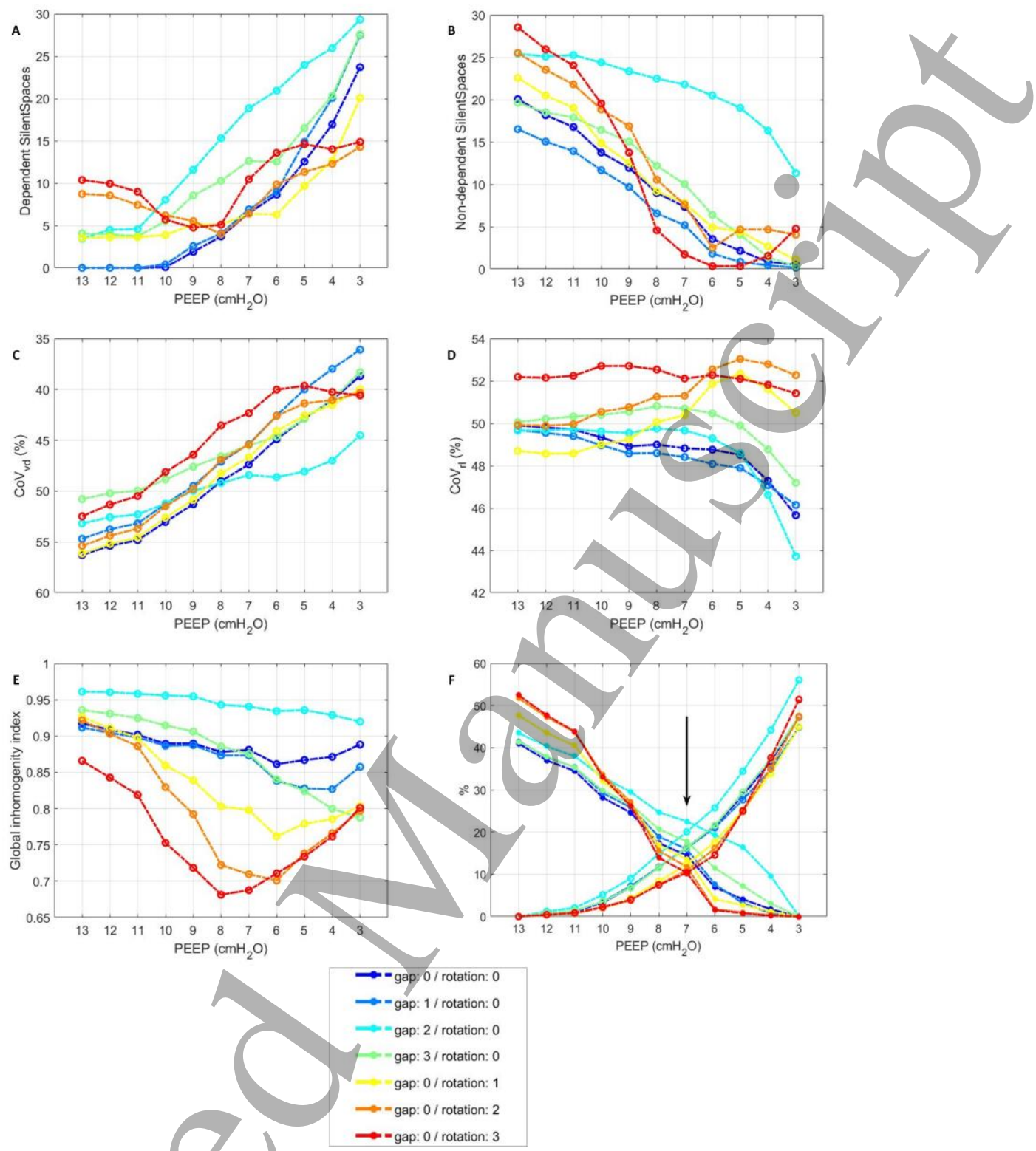

Figure 3 EIT parameters at each different simulated positive end-expiratory pressure (PEEP) levels, gaps and belt rotations. Panels (A) and (B): Dependent silent spaces (DSS) and non-dependent silent spaces (NSS) respectively. Panels (C) and (D) centre of ventilation in the ventrodorsal $\left(\mathrm{CoV}_{\mathrm{vd}}\right)$ and right-to-left $\left(\mathrm{CoV}_{\mathrm{rd}}\right)$ direction. Panel $(\mathrm{E})$ : global inhomogeneity index (GI index). Colours demonstrate the studied gap and belt rotation permutations. Panel (F): Percentages (\%) of lung collapse (CL) and overdistension (OD). OD is represented by closed circles, CL is represented as open circles. The arrow is showing the best compromise PEEP level, i.e. the "optimal PEEP" level with equally balanced OD and CL.

The electrode gap has less of an influence on the EIT parameters. In all three levels of electrode gaps, all EIT parameters except NSS demonstrated similar behaviour as no gap or belt rotation. With an electrode gap of 2 , the NSS were $>10 \%$ even at low PEEP levels.

The absolute percentages of OD/CL vary with different magnitudes of belt rotation and gap (Figure 3). Of note, this does not impede the ability of the approach suggested by Costa et al. (2009) to calculate the "best compromise" PEEP level. A PEEP value of $7 \mathrm{cmH}_{2} \mathrm{O}$ was associated with equally balanced OD and CL for all degrees of the sternal gap and belt rotation. The calculated 
amount of OD/CL at the "optimal PEEP" level varied between 9.3/10.1\% (No Gap, 4 Rot) and 22.6/20.1\% (2 Gap, No Rot).

\section{Clinical results}

Seven infants participated in the study with systematic rotation of the EIT belt (Table 1). The sternal gap could not be adjusted, as this was dependent on the size of the chest circumference and belt used, the mean (SD) sternal gap was $1.5(1.0) \mathrm{cm}$.

Table 1: Subject characteristics.

\begin{tabular}{cccccccc}
\hline $\begin{array}{c}\text { Subject } \\
\text { number }\end{array}$ & $\begin{array}{c}\text { Postnatal } \\
\text { age } \\
\text { (days })\end{array}$ & $\begin{array}{c}\text { Weight } \\
(\mathrm{g})\end{array}$ & $\begin{array}{c}\text { Chest } \\
\text { circumference } \\
(\mathrm{cm})\end{array}$ & $\begin{array}{c}\text { Belt size } \\
(\mathrm{cm})\end{array}$ & $\begin{array}{c}\text { Sternal } \\
\text { gap } \\
(\mathrm{cm})\end{array}$ & $\begin{array}{c}\text { Respiratory Ventilation } \\
\text { diagnosis }\end{array}$ & $\begin{array}{c}\text { mode } \\
\text { mode support }\end{array}$ \\
2 & 0.4 & 2690 & 30.5 & 27.5 & 1.5 & RDS & No support \\
3 & 1.0 & 3630 & 34.0 & 33.0 & 0.5 & Sepsis & No support \\
4 & 0.3 & 4060 & 37.0 & 33.0 & 1.5 & TTN & No support \\
5 & 14.0 & 870 & 21.5 & 20.0 & 1.0 & RDS & nCPAP \\
6 & 27.0 & 1400 & 26.0 & 23.5 & 3.0 & RDS & No support \\
7 & 11.0 & 798 & 20.5 & 20.0 & 0.5 & RDS & PCV \\
\hline Mean & 7.7 & 2281 & 28.4 & --- & $\mathbf{1 . 5}$ & --- & --- \\
SD & 10.3 & 1302 & 6.1 & --- & $\mathbf{1 . 0}$ & --- & --- \\
\hline
\end{tabular}

Abbreviations: RDS; respiratory distress syndrome, TTN; transient tachypnoea of the newborn, nCPAP; nasal continuous positive airway pressure, HFNC; high flow nasal cannula, PCV; pressure-controlled ventilation.

A)

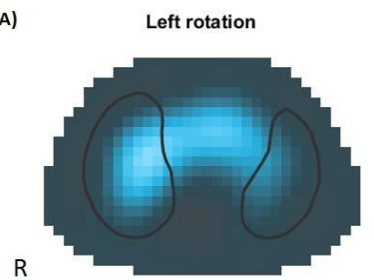

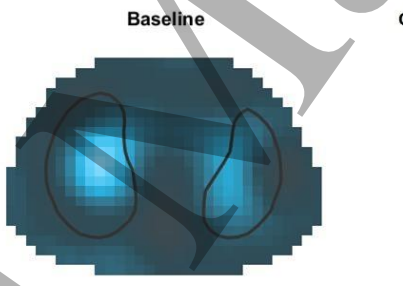

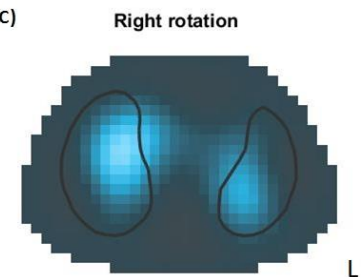

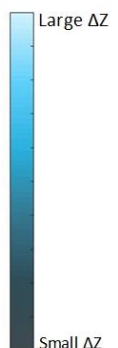

Figure 4. Representative functional EIT images of tidal ventilation with left (A), baseline (B) and right (C) belt rotation for Infant 1. Body chest circumference was $30.5 \mathrm{~cm}$, belt size $27.5 \mathrm{~cm}$. The relative magnitude of tidal ventilation in each region is shown using a heat map with light blue the greatest ventilation and dark blue the least. Lung contours are indicated as black lines. $\mathrm{R}$, right; $\mathrm{L}$, left

Figure 5 shows the DSS, NSS, CoV and GI data for each belt position. Belt rotation significantly altered DSS ( $\mathrm{p}=0.003$; repeated-measure ANOVA), NSS $(\mathrm{p}=0.007)$ and $\mathrm{CoVrl}(\mathrm{p}=0.003)$, but no statistically significant difference was observed for CoVvd $(\mathrm{p}=0.11)$ or GI index $(\mathrm{p}=0.22)$. Belt rotation to the left resulted in a mean $(95 \% \mathrm{CI})$ increase in DSS and NSS of $-5.0(-9.3,-1.0) \%, 3.2$ $(0.1,6.5) \%$ and a $2.2(-0.9,5.2) \%$ decrease in $\mathrm{CoV}_{\mathrm{rl}}$ compared to baseline (Bonferroni post tests). Rightward rotation resulted in a $-4.9(-7.0,-2.7) \%,-0.9(-4.2,2.4) \%$ and $8.4(1.0,15.7) \%$ change from baseline for DSS, NSS and $\mathrm{CoV}_{\mathrm{rl}}$ respectively. 


\section{Discussion}

Figure 5. Change in $\mathrm{CoV}_{\mathrm{vd}}$ (ventrodorsal centre of ventilation), $\mathrm{CoV}_{\mathrm{rd}}$ (right-to-left centre of ventilation), GI (global inhomogeneity index), DSS (dependent Silent Spaces) and NSS (non-dependent Silent Spaces) EIT parameters from baseline (no rotation) values during $2 \mathrm{~cm}$ left (red) and right (green) belt rotation. Boxes represent $25^{\text {th }}-75^{\text {th }}$ percentiles and median (whiskers $5^{\text {th }}$ and $95^{\text {th }}$ confidence interval). Asterisks indicate the outliers. $\mathrm{p}<0.05$ difference compared to baseline (repeated-measures ANOVA with Bonferroni post-test).

Most neonatal EIT studies have been performed with 16 individual adhesive ECG electrodes (Frerichs et al 2003, Dunlop et al 2006, Riedel et al 2009, Miedema et al 2011, 2013), but in more recent studies (Sophocleous et al, Khodadad et al 2018) and case reports (Miedema et al 2017, Oliva et al 2017, Tingay et al 2019b, Rahtu et al 2019), a novel neonatal EIT textile belt with 32 non-adhesive electrodes has been used. While the EIT belt simplifies the placement of electrodes on the patient's chest, the impact of the sternal gap caused by the limited number of available EIT belts sizes, and the rotation of the electrodes due to movement of the patient have not been previously studied in detail. As the validity and reproducibility of monitoring results are of great importance, we consider the evaluation of the impact of these conditions on pulmonary EIT parameters in neonatal monitoring applications (Frerichs and Becher 2019) as crucial.

In this study we developed a simulation framework, based on a three-dimensional finite element model, introduced lung regions with little or no ventilation and changed them according to a decremental PEEP trial. The sternal gap and belt rotation were then simulated and their influence on commonly used EIT parameters was studied. Assuming the ideal situation of no sternal gap and no belt rotation, the model showed the expected behaviour; with open dorsal lung areas at high PEEP levels and stepwise lung collapse with PEEP reduction (Zhao et al 2019, Gattinoni et al 2017). The opposite behaviour was observed in the ventral gravity non-dependent lung areas as expected.

Although differences between the optimal condition, the different combinations of gap distances and belt rotations could be seen on all EIT parameters, the overall behaviour of different lung regions remained as expected over the whole range of PEEP changes. DSS and NSS were found to be insensitive to sternal gaps with a single electrode distance rotation, but this was not the case for 
belt rotations of 2 and 3 electrode spaces. This can be observed in the tidal images at these large belt rotations (Figure 2). The confounding effect of greater than one electrode space belt rotation should be taken into consideration when interpreting tidal images obtained under such circumstances.

While a belt rotation of several electrodes is rather unexpected in adult patients in an intensive care environment it is more likely to be observed in neonatal and paediatric patients, particularly older, un-sedated, infants. In our current study, we showed the influence of leftward and rightward belt rotation (Figure 4) on lung EIT parameters in seven infants treated in NICUs. Since the gap was introduced from the beginning of the measurement, due to a limited number of different belt sizes, the effect of the gap was not evaluated. In very premature infants with a thorax circumference of less than $30 \mathrm{~cm}$, two-electrode displacement is less than $2 \mathrm{~cm}$ with the EIT belts used in this study. The clinical results were consistent with the simulation data, although some EIT parameters were less sensitive to belt rotation in the small group of infants we studied. The discrepancies between the changes in EIT parameters following left and right rotation are probably related to the anatomical differences between the right and left lung. Similar asymmetry was also observed by Braun et al (2018). In general, $\mathrm{CoV}_{\mathrm{vd}}$ and GI index seem to be more robust to the rotational belt displacements examined in our study, than the other measures. This is reassuring as they also represent commonly reported parameters of ventilation inhomogeneity in the clinical EIT setting (Frerichs et al 2017) and considered highly important in neonatal/paediatric EIT applications (Frerichs and Becher 2019).

PEEP trial analysis revealed that the absolute percentages of OD and CL, calculated according to the Costa et al (2009) approach were affected by belt rotation and gap, but the approach could identify the optimal PEEP level with all degrees of rotation and gap investigated. This is important for the clinical users of current EIT technology as the robustness of the applied PEEP optimisation approach is demonstrated.

We suggest that a belt rotation detection algorithm with automatic compensation (Boyle et al 2017) should be implemented when conducting routine EIT monitoring of neonates in whom handling should be minimised. The development of EIT belts with shoulder straps, as previously described for adult use (Waldmann et al 2017), might prove valuable as a method of minimising belt rotation in patients of younger age as well.

Although three-dimensional EIT does not play any role in the current clinical use of EIT this might change in the future. Therefore, belt rotations as described in our study should be taken into account in three-dimensional EIT applications because they will inevitably result in electrode placement errors (Graham and Adler 2007).

The present work is limited in that the systematic belt rotation was performed on only seven infants in the NICUs. However, the combination of this clinical data with the theoretical simulation data rendered the described effects of electrode gap and belt rotations robust.

Our study shows that either minimising right or leftward belt rotation, or adapting for it in image reconstruction will be an important challenge for EIT applications in neonates. We demonstrated that the small violation of the equidistance criterion between the first and the last electrode of the electrode array and rotations of the belt less than 1 electrode space, exert only minor effects on EIT parameters and are not expected to impede the interpretation. However, rotations of a slightly higher degree, greater than one electrode space in the present study, induce non-negligible effects on the calculated EIT parameters that might lead to flawed interpretations. Therefore, the clinical users of current EIT technology should be instructed to minimise the incidence of belt rotations, especially in neonatal applications. Algorithms detecting belt rotation with implemented automatic compensation and further refinement of EIT belt designs could also be considered. 


\section{Conclusion}

In this paper we investigated the effect of sternal electrode gap and belt rotation on the robustness of commonly used lung EIT parameters and we make the following observations:

- While commonly described EIT parameters are rather insensitive to the electrode gap at the sternum, they are influenced by rotational belt displacement.

- In neonatal patients with a thorax circumference of less than $30 \mathrm{~cm}$, belt rotation should be taken into consideration and a sufficient number of belts with fine length gradations should be provided.

- Automatic detection of belt rotation with implemented compensation in image reconstruction and/or further refinement of EIT belt designs might be beneficial.

\section{Acknowledgments}

This project has received funding from the European Union's Horizon 2020 Research and Innovation Programme under the grant agreement No. 668259. Dr. Tingay is supported by a National Health and Medical Research Council Clinical Career Development Fellowship (Grant ID 11123859) and the Victorian Government Operational/Infrastructure Support Program (Melbourne, Australia).

\section{References}

Adler A, Amato M B, Arnold J H, Bayford R, Bodenstein M, Böhm S H, Brown B H, Frerichs I, Stenqvist O, Weiler N and Wolf G K 2012 Whither lung EIT: Where are we, where do we want to go and what do we need to get there? Physiol. Meas. 33 679-94

Adler A, Arnold J H, Bayford R, Borsic A, Brown B, Dixon P, Faes T J C, Frerichs I, Gagnon H, Gärber Y, Grychtol B, Hahn G, Lionheart W R B, Malik A, Patterson R P, Stocks J, Tizzard A, Weiler N and Wolf G K 2009a GREIT: a unified approach to 2D linear EIT reconstruction of lung images Physiol. Meas. 30 S35-55

Barber D C and Brown B H 1988 Errors in reconstruction of resistivity images using a linear reconstruction technique Clin. Phys. Physiol. Meas. 9 101-4

Bayford R H 2006 Bioimpedance Tomography (Electrical Impedance Tomography) Annu. Rev. Biomed. Eng. 8 63-91

Blankman P, Hasan D, Erik G J and Gommers D 2014 Detection of "best" positive endexpiratory pressure derived from electrical impedance tomography parameters during a decremental positive end-expiratory pressure trial Crit. Care 18 261-6

Boyle A, Crabb M G, Jehl M, Lionheart W R B and Adler A 2017 Methods for calculating the electrode position Jacobian for impedance imaging Physiol. Meas. 38 555-74

Braun F, Proença M, Lemay M, Bertschi M, Adler A, Thiran J P and Solà J 2018 Limitations and challenges of EIT-based monitoring of stroke volume and pulmonary artery pressure Physiol. Meas. 39014003

Brown B H, Barber D C and Seagar A D 1985 Clinical Physics and Physiological Measurement Applied potential tomography: possible clinical adpplications Clin. Phys. Physiol. Meas. Physiol. Meas 6 109-21

Chatziioannidis I, Samaras T and Nikolaidis N 2011 Electrical impedance tomography: A new study method for neonatal respiratory distress syndrome? Hippokratia 15 211-5

Costa E L V, Borges J B, Melo A, Suarez-Sipmann F, Toufen C, Bohm S H and Amato M B P 2012 Bedside estimation of recruitable alveolar collapse and hyperdistension by electrical impedance tomography Appl. Physiol. Intensive Care Med. 1 Physiol. Notes - Tech. Notes Semin. Stud. Intensive Care, Third Ed. 165-70 
Costa E L V, Borges J B, Melo A, Suarez-Sipmann F, Toufen C, Bohm S H and Amato M B P 2009 Bedside estimation of recruitable alveolar collapse and hyperdistension by electrical impedance tomography Intensive Care Med. 35 1132-7

Dunlop S, Hough J, Riedel T, Fraser J F, Dunster K and Schibler A 2006 Electrical impedance tomography in extremely prematurely born infants and during high frequency oscillatory ventilation analyzed in the frequency domain. Physiol. Meas. 27 1151-65

Frerichs I, Amato M B P, van Kaam A H, Tingay D G, Zhao Z, Grychtol B, Bodenstein M, Gagnon H, Böhm S H, Teschner E, Stenqvist O, Mauri T, Torsani V, Camporota L, Schibler A, Wolf G K, Gommers D, Leonhardt S, Adler A and TREND study group 2017 Chest electrical impedance tomography examination, data analysis, terminology, clinical use and recommendations: consensus statement of the TRanslational EIT developmeNt stuDy group. Thorax 72 83-93

Frerichs I and Becher T 2019 Chest electrical impedance tomography measures in neonatology and paediatrics-a survey on clinical usefulness Physiol. Meas. 40054001

Frerichs I, Dargaville P A, Van Genderingen H, Morel D R and Rimensberger P C 2006 Lung volume recruitment after surfactant administration modifies spatial distribution of ventilation Am. J. Respir. Crit. Care Med. 174 772-9

Frerichs I, Hahn G and Hellige G 1999 Thoracic electrical impedance tomographic measurements during volume controlled ventilation-effects of tidal volume and positive end-expiratory pressure IEEE Trans. Med. Imaging 18 764-73

Frerichs I, Schiffmann H, Hahn G and Hellige G 2001 Non-invasive radiation-free monitoring of regional lung ventilation in critically ill infants. Intensive Care Med. 27 1385-94

Frerichs I, Schiffmann H, Oehler R, Dudykevych T, Hahn G, Hinz J and Hellige G 2003 Distribution of lung ventilation in spontaneously breathing neonates lying in different body positions Intensive Care Med 29 787-94

Gabriel C, Gabriel S and Corthout E 1996 The dielectric properties of biological tissues: I. Literature survey. Phys. Med. Biol. 41 2231-49

Gabriel C, Peyman A and Grant E H 2009 Electrical conductivity of tissue at frequencies below 1 MHz Phys. Med. Biol. 54 4863-78

Gattinoni L, Collino F, Maiolo G, Rapetti F, Romitti F, Tonetti T, Vasques F and Quintel M 2017 Positive end-expiratory pressure: how to set it at the individual level. Ann. Transl. Med. 5 288

Graham B M and Adler A 2007 Electrode placement configurations for 3D EIT Physiol. Meas. 28 28-44

Grychtol B and Adler A 2014 Choice of reconstructed tissue properties affects interpretation of lung EIT images. Physiol. Meas. 35 1035-50

Grychtol B and Adler A 2013 Uniform background assumption produces misleading lung EIT images. Physiol. Meas. 34 579-93

Hampshire A 1995 Multifrequency and parametric EIT images of neonatal lungs Physiol. Meas 16

Hough J, Trojman A and Schibler A 2016 Effect of time and body position on ventilation in premature infants Pediatr. Res. 80 499-504

Kallio M, van der Zwaag A-S, Waldmann A D, Rahtu M, Miedema M, Papadouri T, van Kaam A H, Rimensberger P C, Bayford R and Frerichs I 2019 Initial Observations on the Effect of Repeated Surfactant Dose on Lung Volume and Ventilation in Neonatal Respiratory Distress Syndrome. Neonatology 1-5

Karsten J, Grusnick C, Paarmann H, Heringlake M and Heinze H 2015 Positive end-expiratory pressure titration at bedside using electrical impedance tomography in post-operative cardiac surgery patients Acta Anaesthesiol. Scand. 59 723-32

Karsten J, Stueber T, Voigt N, Teschner E and Heinze H 2016 Influence of different electrode

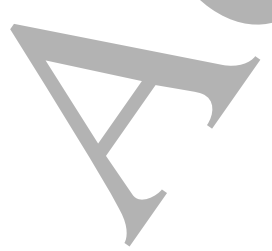


belt positions on electrical impedance tomography imaging of regional ventilation: A prospective observational study Crit. Care 20 1-10

Khodadad D, Nordebo S, Müller B, Waldmann A, Yerworth R, Becher T, Frerichs I, Sophocleous L, Van Kaam A, Miedema M, Seifnaraghi N and Bayford R 2018 Optimized breath detection algorithm in electrical impedance tomography Physiol. Meas. 39

Krueger-Ziolek S, Schullcke B, Kretschmer J, Müller-Lisse U, Möller K and Zhao Z 2015 Positioning of electrode plane systematically influences EIT imaging Physiol. Meas. 36 1109-18

Lozano A, Rosell J and Pallás-Areny R 1995 Errors in prolonged electrical impedance measurements due to electrode repositioning and postural changes. Physiol.Meas. $16121-$ 30

Luepschen H, Meier T, Grossherr M, Leibecke T, Karsten J and Leonhardt S 2007 Protective ventilation using electrical impedance tomography Physiol. Meas. 28

McCall K E, Waldmann A D, Pereira-Fantini P, Oakley R, Miedema M,Perkins E J, Davis P G, Dargaville P A, Böhm S H, Dellacà R, Sourial M, Zannin E, Rajapaksa A E, Tan A, Adler A, Frerichs I and Tingay D G 2017 Time to lung aeration during a sustained inflation at birth is influenced by gestation in lambs Pediatr. Res. 82 712-20

Miedema M, van der Burg P S, Beuger S, de Jongh F H, Frerichs I and van Kaam A H 2013 Effect of nasal continuous and biphasic positive airway pressure on lung volume in preterm infants. J. Pediatr. 162 691-7

Miedema M, de Jongh F H, Frerichs I, van Veenendaal M B and van Kaam A H 2011 Changes in lung volume and ventilation during surfactant treatment in ventilated preterm infants. Am.J. Respir. Crit. Care Med. 184 100-5

Miedema M, de Jongh F H, Frerichs I, van Veenendaal M B and van Kaam A H 2012 Regional respiratory time constants during lung recruitment in high-frequency oscillatory ventilated preterm infants. Intensive Care Med. 38 294-9

Miedema M, Waldmann A, McCall K E, Böhm S H, van Kaam A H and Tingay D G 2017 Individualized Multiplanar Electrical Impedance Tomography in Infants to Optimize Lung Monitoring. Am. J. Respir. Crit. Care Med. 195 536-8

Oliva P de la, Waldmann A D, Böhm S H, Verdú-Sánchez C, Pérez-Ferrer A and Alvarez-Rojas E 2017 Bedside Breath-Wise Visualization of Bronchospasm by Electrical Impedance Tomography Could Improve Perioperative Patient Safety: A Case Report A A Case Reports $81-4$

Rabbani K S and H.Kabir A M B 1991 Studies on the effect of the third dimension on a twodimensional electrical impedance tomography system Clin. Phys. Physiol. Meas. 12 393402

Rahtu M, Frerichs I, Waldmann A D, Strodthoff C, Becher T, Bayford R and Kallio M 2019 Early Recognition of Pneumothorax in Neonatal RDS with Electrical Impedance Tomography Am. J. Respir. Crit. Care Med. rccm.201810-1999IM

Reifferscheid F, Elke G, Pulletz S, Gawelczyk B, Lautenschläger I, Steinfath M, Weiler N and Frerichs I 2011 Regional ventilation distribution determined by electrical impedance tomography: Reproducibility and effects of posture and chest planeresp Respirology 16 $523-31$

Riedel T, Kyburz M, Latzin P, Thamrin C and Frey U 2009 Regional and overall ventilation inhomogeneities in preterm and term-born infants Intensive Care Med. 35 144-51

Rocha G, Soares P, Gonçalves A, Silva A I, Almeida D, Figueiredo S, Pissarra S, Costa S, Soares H, Flôr-De-Lima F and Guimarães H 2018 Respiratory Care for the Ventilated Neonate

Sobota V and Roubik K 2016 Center of Ventilation-Methods of Calculation Using Electrical Impedance Tomography and the Influence of Image Segmentation (Springer, Cham) pp $1264-9$ 
Sophocleous L, Frerichs I, Miedema M, Kallio M, Papadouri T, Karaoli C, Becher T, Tingay D G, van Kaam A, Bayford R H and Waldmann A D 2018 Clinical performance of a novel textile interface for neonatal chest electrical impedance tomography Physiol. Meas. 39 04004

Spadaro S, Mauri T, Böhm S H, Scaramuzzo G, Turrini C, Waldmann A D, Ragazzi R, Pesenti A and Volta C A 2018 Variation of poorly ventilated lung units (silent spaces) measured by electrical impedance tomography to dynamically assess recruitment Crit. Care 22 1-9

Taktak A, Record P, Gadd R and Rolfe P 1995 Practical factors in neonatal lung imaging using electrical impedance tomography Med. Biol. Eng. Comput. 33 202-5

Tang M, Wang W, Wheeler J, McCormick M and Dong X 2002 Effects of incompatible boundary information in EIT on the convergence behavior of an iterative algorithm IEEE Trans. Med. Imaging 21 620-8

Tingay D G, Lavizzari A, Zonneveld C E E, Rajapaksa A E, Zannin E, Perkins E, Black D, Sourial M, Dellacà R L, Mosca F, Adler A, Grychtol B, Frerichs I and Davis P G 2015 An Individualized Approach To Sustained Inflation Duration At Birth Improves Outcomes In Newborn Preterm Lambs Am. J. Physiol. - Lung Cell. Mol. Physiol. ajplung.00277.2015

Tingay D G, Pereira-Fantini P M, Oakley R, McCall K E, Perkins E J, Miedema M, Sourial M, Thomson J, Waldmann A, Dellaca R L, Davis P G and Dargaville P A 2019a Gradual Aeration at Birth is More Lung Protective than a Sustained Inflation in Preterm Lambs Am. J. Respir. Crit. Care Med. rccm.201807-1397OC

Tingay D G, Waldmann A D, Frerichs I, Ranganathan S and Adler A 2019b Electrical Impedance Tomography Can Identify Ventilation and Perfusion Defects: A Neonatal Case Am. J. Respir. Crit. Care Med. 199 384-6

Ukere A, März A, Wodack K H, Trepte C J, Háese A, Waldmann A D, Böhm S H and Reuter D A 2016 Perioperative assessment of regional ventilation during changing body positions and ventilation conditions by electrical impedance tomography ed T Asai Br. J. Anaesth. 117 $228-35$

Waldmann A D, Wodack K H, März A, Ukere A, Trepte C J, Böhm S H and Reuter D A 2017 Performance of Novel Patient Interface for Electrical Impedance Tomography Applications J. Med. Biol. Eng. 37 561-6

Zhao Z, Chang M Y, Chang M Y, Gow C H, Zhang J H, Hsu Y L, Frerichs I, Chang H T and Möller K 2019 Positive end-expiratory pressure titration with electrical impedance tomography and pressure-volume curve in severe acute respiratory distress syndrome Ann. Intensive Care 97

Zhao Z, Möller K, Steinmann D, Frerichs I and Guttmann J 2009 Evaluation of an electrical impedance tomography-based global inhomogeneity index for pulmonary ventilation distribution Intensive Care Med. 35 1900-6 